\title{
Measuring the accuracy of softball impact simulations
}

\author{
Lloyd Smith $^{1} \cdot$ Derek Nevins ${ }^{1} \cdot$ Ngo Tien Dat $^{2} \cdot$ Pascal Fua $^{2}$
}

Published online: 21 May 2016

(C) International Sports Engineering Association 2016

\begin{abstract}
A study has been conducted to review viscoelastic and foam-constitutive models to describe sport ball response to impact with a rigid cylindrical surface. The impact model was developed to simulate a ball-bat collision. Comparisons were made to actual impacts, utilizing displacements recorded and analyzed using high-speed video capture. The resulting images and the ball geometry before impact were used as input to a computer-vision algorithm, which then produced a quantitative description of the deformation during impact. Foam-based material models were observed to match this observed deformation better (within 1\%) than viscoelastic material models (within $5 \%$ ). Both viscoelastic and foam material models deviated more from experimental data when describing dissipated energy and stiffness than when describing deformation. When describing impact energy dissipation and ball stiffness, the viscoelastic models deviated from experiment by more than a factor of two, while the foam material models exhibited up to $35 \%$ error. The measured ball deformation, afforded through video analysis, has shown that foam material models are better able to describe ball impacts involving large energy dissipation, but require further refinement before equipment performance and design can be reliably performed.
\end{abstract}

Lloyd Smith

lvsmith@wsu.edu

1 Washington State University, Pullman, WA, USA

2 École Polytechnique Fédérale, Lausanne, Switzerland

\section{Introduction}

Sport ball impact modeling is among the most challenging events to capture numerically. It is a contact problem, where the impact area is a function of force magnitude. Ball impact forces are usually large and of short duration, requiring a solution with high temporal fidelity. Balls are typically made from time-dependent materials, making the solution rate dependent. The ball deformation is usually large, requiring the use of finite strain formulations. Many ball materials exhibit non-linear response, requiring a nonlinear material constitutive relation. An accurate description of sports balls is needed, nevertheless, to design protective equipment, such as helmets and pads, and striking equipment, such as bats, clubs and rackets.

Given the geometric and material non-linearities inherent to ball impacts, finite element analysis is often used to simulate them. Numerous material models have been investigated, including elastic [1], rubber [2], viscoelastic $[3,4]$ and foam [5]. The numerical models are typically calibrated and compared against instrumented ball impacts [6], although examples involving material characterization can also be found $[5,7]$.

There is a need to compare impact simulations with empirical results to assess which predictions most accurately describe a physical quantity of interest. For example, when testing protective equipment or durability, peak impact force is key. By contrast, when testing for equipment performance in the case of bat, club or racket impacts, energy dissipation matters most.

The solution to numerical impact problems are not single valued. It is possible, for instance, to simultaneously show good agreement with impact force or energy dissipation, and poor agreement with ball deformation or contact duration. In this scenario, a ball model may show good 
agreement with instrumented calibration tests, but perform differently from the physical ball when the impact geometry is changed [4]. To overcome this problem, we propose using a video-based reconstruction technique to describe the ball shape during instrumented impacts. The results are compared with those of finite element simulations to consider the ability of different material models to describe ball deformation as well as peak force and coefficient of restitution during impact.

\section{Methodology}

The ball used for this work was a solid adult slow-pitch softball measuring $300 \mathrm{~mm}$ in circumference and weighing $190 \mathrm{~g}$ (A9044, Wilson). Softballs are made of rigid closed cell, high-density polyurethane $\left(0.38 \mathrm{~g} / \mathrm{cm}^{3}\right)$ foam with a $1.5 \mathrm{~mm}$-thick leather covering.

\section{Impact testing}

As the polyurethane is sensitive to moisture and temperature, the test ball was preconditioned at $22{ }^{\circ} \mathrm{C}$ and $50 \%$ $\mathrm{RH}$ for 14 days. The conditioned test ball was weighed (m) and impacted at $42.5 \mathrm{~m} / \mathrm{s}$ against a rigidly mounted solid half-cylinder of $57 \mathrm{~mm}$ diameter, as shown in Fig. 1. Three piezoelectric load cells (208C03, PCB) were placed between the half-cylinder and rigid wall to measure the impact force [8]. The load signals were summed and the

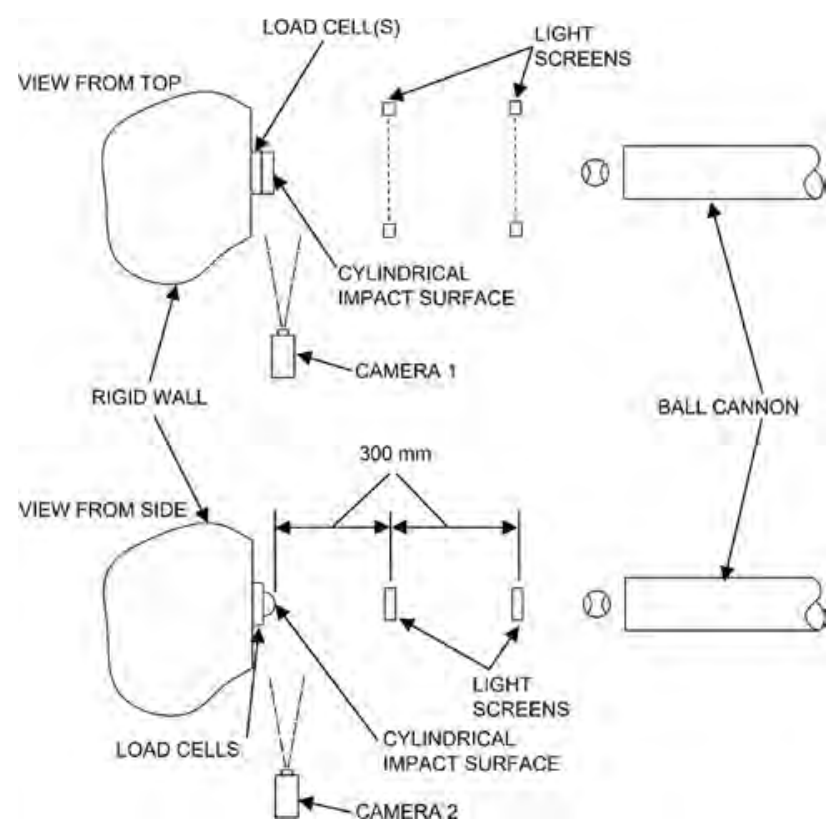

Fig. 1 Apparatus used to measure ball impact response (above: top view, below: side view) force-time response was measured at a frequency of $100 \mathrm{kHz}$ (temporal resolution of 10 microseconds). The inbound $\left(v_{i}\right)$ and rebound $\left(v_{r}\right)$ ball velocities were measured using light screens placed between the cannon and impact surface. The ratio of the rebound to inbound speed was used to obtain the coefficient of restitution for each impact. By equating the kinetic energy of the incoming ball of speed, $v_{i}$, with the stored energy at maximum deformation, with a force, $F$, an expression for the ball stiffness, $k$, may be obtained as [8]

$k=\frac{1}{m}\left(\frac{F}{v_{i}}\right)^{2}$.

The test ball was impacted four times prior to testing to "break-in" the ball and avoid potential softening effects. The ball was projected four times at $42.5 \mathrm{~m} / \mathrm{s}$, from which a representative impact was selected for comparison with video reconstruction and the finite element models. The ball was rested for 2 min between impacts to minimize frictional heating effects on the ball's response.

\subsection{Video analysis}

Ball impacts were recorded using two high-speed video cameras. While two-camera video reconstruction is often used to capture the motion of discrete points [9-11], the aim of this work was to explicitly reconstruct the ball shape in 3D space. Camera \#1 was aligned with the long axis of the impact cylinder, while camera \#2 was mounted above it (Fig. 1). The cameras recorded the impact at 13,000 frames per second with an exposure time of $15 \mu$ s and a resolution of 704 by 704 pixels. Prior to impact, a checkered board was rotated through several positions within the capture volume for camera calibration. The 3D shape of the deforming ball was determined using image cues from both views according to a technique known as template-based reconstruction [12-14]. As illustrated in Fig. 2, the deformed shape can be defined by inferring the 3D shape of a surface from the input images, given a reference image in which the 3D undeformed shape is known. The algorithm establishes correspondences between surface points in the template and input images; that is points that are the projections of the same 3D points, but have shifted due to surface deformation. From these shifts, the 3D deformation is inferred as described in detail in [15].

In the case of the ball depicted in Fig. 2, we take the 3D template to be a simple sphere with the appropriate radius and the reference image to be an image of the ball before impact. The correspondences are mostly established between the projections of points corresponding to the ball markings. Silhouette, symmetry and impact surface nonpenetration constraints are enforced to yield results, such as 


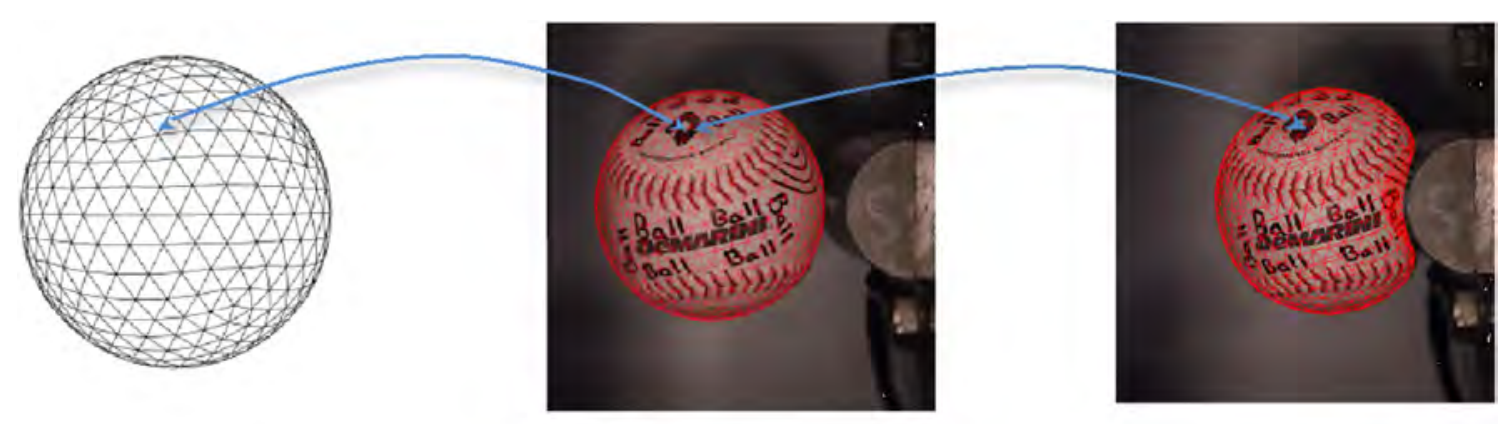

(a)

(b)

(c)

Fig. 2 Template-based 3D shape reconstruction from camera \#1. a 3D template reconstruction of the undeformed ball shape, $\mathbf{b}$ template overlaid on video capture of undeformed ball, $\mathbf{c}$ template overlaid on video capture of deformed ball. Arrow indicates mapping of surface points between template and video capture
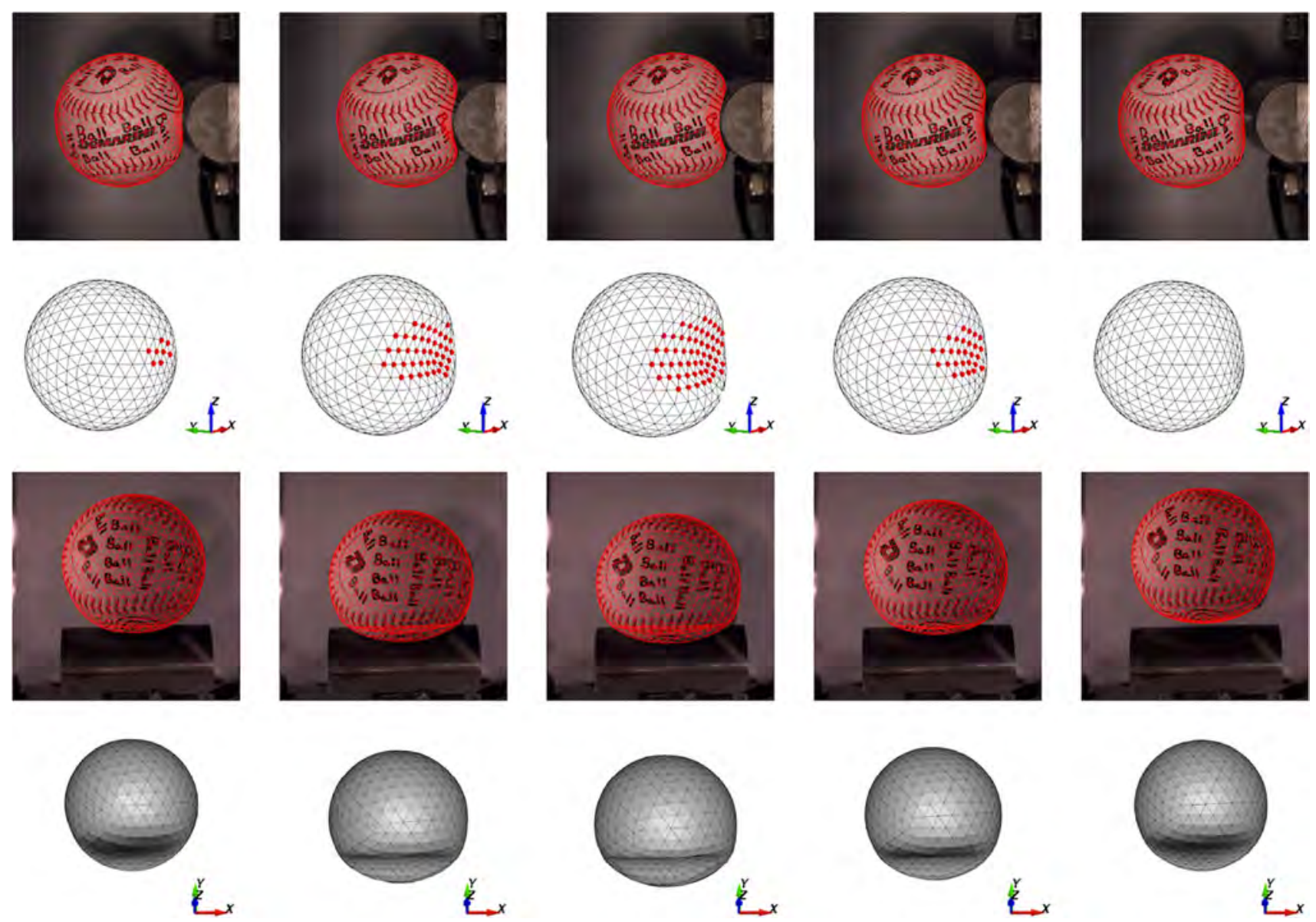

Fig. 3 3D reconstruction of the ball colliding with the cylinder. First and third rows Reprojection of the reconstructed mesh in different frames of the sequence in the side (camera \#1) and top views (camera \#2). Second and fourth rows the reconstructed meshes seen from

those depicted in Fig. 3 The deformed shapes were first computed using a single camera and validated using a second independent camera. The median error on the location of the reconstructed $3 \mathrm{D}$ vertices during the impact different viewpoints, rotated slightly about the $y$ and $x$ axes, respectively, to show the impact surface. The red dots denote vertices touching the cylinder (color figure online)

was of the order $0.6 \mathrm{~mm}$ or less [15]. Both video captures were used simultaneously to maximize the accuracy, and the resultant imagery was compared with the FEA simulations. 


\subsection{Finite element analysis}

The softball (cover and core) was modeled as a homogeneous sphere. This approximation is justified on a number of grounds. First, the contribution of the $1.5 \mathrm{~mm}$ leather cover to the ball's response is small. Balls tested with and without covers differed in their coefficient of restitution and stiffness by less than 1 and $5 \%$, respectively. Second, the modulus of leather is an order of magnitude smaller than polyurethane. The low modulus and small thickness of the cover explain its small contribution to the ball's response. Third, the aim of this work was to compare the measured ball response with numerical simulations. Thus, separate characterization of the cover and core materials was not needed.

Ball models were developed in the LS-DYNA finite element code (Version 971 revision 6, LSTC, Livermore, CA). The balls were modeled using 7168 eight-noded fully integrated solid brick elements. The solid steel cylinder was modeled with 4864 eight-noded reduced integration solid brick elements. A convergence study was conducted on a similar model [5]. When the number of elements was doubled, the impulse, displacement and peak impact force changed only by $2 \%$. Further refinement was, therefore, not considered. A "surface to surface" contact was defined between the objects to prevent penetration during impact. The model included two symmetry planes to reduce solution time. The ball was modeled with a density of $0.417 \mathrm{~g} /$ $\mathrm{cm}^{3}$ to account for the combined mass of the polyurethane core and leather cover, and an initial velocity of $42.5 \mathrm{~m} / \mathrm{s}$.

Ball response was described using one of four material models. A linear viscoelastic model (MAT 006) of the form

$G(t)=G_{i}+\left(G_{o}-G_{i}\right) e^{-t}$

was employed, where $G, G_{o}$ and $G_{i}$ are the time-dependent, initial and long-term shear moduli, respectively, $t$ is time and $\beta$ described the rate sensitivity. Three sets of coefficients for this model were taken from [14, 16, 17].

A low-density foam material model (MAT 057) was used. Its behavior is dominated by uniaxial loading and assumed to not significantly couple in the transverse directions. The material model is from Storakers and was modified to include hysteretic unloading as described by Chang [17]. For this material model, the compressive load response is defined by a compressive loading curve, a DAMP factor and viscous coefficient. Tensile response is described by a tensile modulus. Unloading is defined by a hysteretic unloading coefficient (HU) and the SHAPE factor. The coefficients for this model were taken from [5].

A medium-density foam material model (MAT 083) was used. The constitutive response was found following the work by Fu-Chang [18] where the hysteretic unloading is a function of the rate sensitivity [5]. The unloading response, $\sigma_{u}$, is determined from the loading response, $\sigma_{L}$, using the following damage formulation for principle stresses:

$\sigma_{u}=(1-d) \sigma_{L}$,

$d=(1-\mathrm{HU})\left(1-\left(\frac{W_{\mathrm{cur}}}{W_{\max }}\right)^{\mathrm{SHAPE}}\right)$,

where $W_{\text {cur }}$ is the current hyperelastic energy per unit deformed volume. The coefficients used for this model were taken from [5].

A fourth material model (MAT 181) used for this work was a hyperelastic model defined from uniaxial loading curves at discrete strain rates, with compressible foam behavior [19]. The coefficients used for this model were found in this work in the following way. A trial compressive stress-strain response was obtained from foam impact tests as described elsewhere [5]. The material model was created using an optimization scheme in LS-DYNA. The trial stress-strain response of a ball impact model was iteratively scaled to maximize agreement with an experimentally measured ball force-displacement response [20, 21]. The scaling factors were relatively large (1.95 and 0.375 for strain and stress, respectively). Likewise, parameters in the MAT 181 material model (damping coefficient, MU; shear modulus; limit stress for damping, SIGP; and Poisson's Ratio, PR) were adjusted during the same optimization scheme to achieve agreement with experimental unloading response of the ball. Thus, the model is essentially a phenomenological fit to the measured response of an instrumented impact.

To match play conditions, the models were trained at $42.5 \mathrm{~m} / \mathrm{s}$, so that agreement with experiment may degrade at other speeds. The ball models were trained from a different ball than that used for video analysis. In this way, neither the viscoelastic ball models nor the foam models were trained on data from the specific ball or impacts used for video reconstruction. The parameters for all the material models are summarized in Table 1 .

Outputs from the finite element models include the load-displacement curve $(L-d)$, coefficient of restitution (e) and stiffness $(k)$, ball diameters $\left(D_{x}, D_{y}, D_{z} ;\right.$ Fig. 4$)$ and ball volume. Load-displacement was obtained by plotting the contact force between the ball and cylinder against displacement of the center node of the ball. Coefficient of restitution was defined as the ratio of the mean velocity of the center node of the ball over the last ten time steps to the initial ball velocity. Stiffness was found using Eq. (1).

In the apparatus described in Fig. 1, dividing the maximum impact force by the ball mass produces the ball acceleration. The ball acceleration is integrated twice, with respect to time, to obtain the ball center of mass displacement, $d$, as 
Table 1 Material parameters for material models used in the finite element simulations of ball impact

\begin{tabular}{|c|c|c|c|c|c|c|c|}
\hline Material model & $\rho\left(\mathrm{g} / \mathrm{cm}^{3}\right)$ & $K(\mathrm{MPa})$ & & $G_{o}(\mathrm{MPa})$ & $G_{i}(\mathrm{MPa})$ & $\beta(\mathrm{Hz})$ & Source \\
\hline MAT 006a & 0.417 & 68,900 & & 68.9 & 4.83 & 68,000 & {$[4]$} \\
\hline MAT 006b & 0.417 & 689 & & 138 & 6.89 & 68,000 & {$[17]$} \\
\hline \multirow[t]{2}{*}{ MAT 006c } & 0.417 & 6890 & & 24.1 & 2.76 & 40,000 & [16] \\
\hline & $\rho\left(\mathrm{g} / \mathrm{cm}^{3}\right)$ & $E(\mathrm{MPa})$ & & $\mathrm{HU}$ & SHAPE & DAMF & \\
\hline MAT 057 & 0.417 & 138 & & 0.01 & 6 & 1.8 & [5] \\
\hline \multirow[t]{2}{*}{ MAT 083} & 0.417 & 138 & & 0.01 & 0.57 & 5.5 & [5] \\
\hline & $\rho\left(\mathrm{g} / \mathrm{cm}^{3}\right)$ & $K(\mathrm{MPa})$ & MU & $G(\mathrm{MPa})$ & SIGF & & \\
\hline MAT 181 & 0.417 & 138 & 0.75 & 717 & 1.79 & & - \\
\hline
\end{tabular}

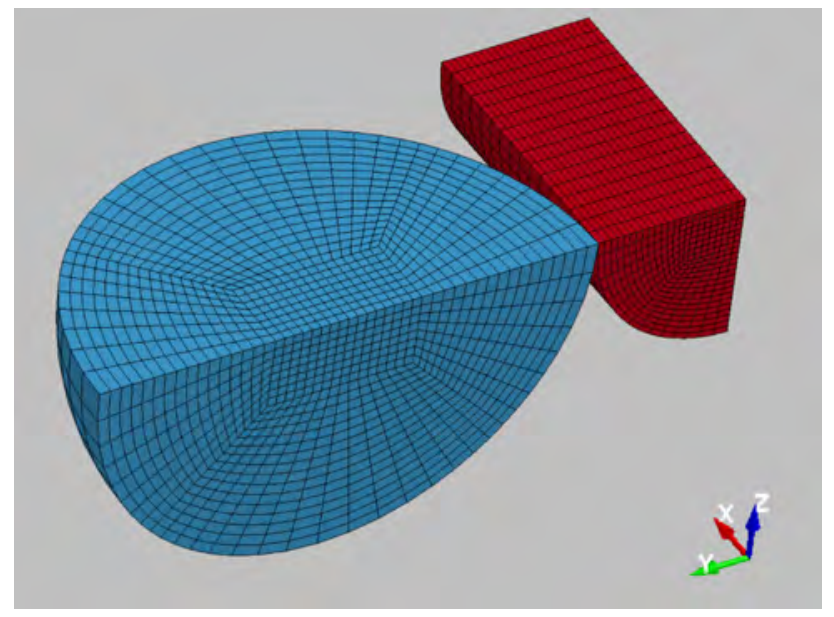

Fig. 4 Views of a mesh of a softball impacting a solid cylinder, showing two planes of symmetry

$d(t)=\int_{0}^{T}\left(v_{i}-\int_{0}^{T} \frac{F(t)}{m} d t\right) d t$.

The experimental ball force-displacement response was obtained in this manner to compare with the numerical models.

\section{Results}

Figure 5 compares the experimental load-displacement response with the numeric models. The curves show large hysteresis, necessary to describe large energy dissipation and a relatively small coefficient of restitution, characteristic of a softball. Given the material and geometric nonlinearities present in ball impacts, it is perhaps surprising that the experimental loading response is nearly linear. The unloading response is also nearly linear (but not elastic), showing a persistent deformation at zero load (that recovers within a few seconds after impact). The numeric

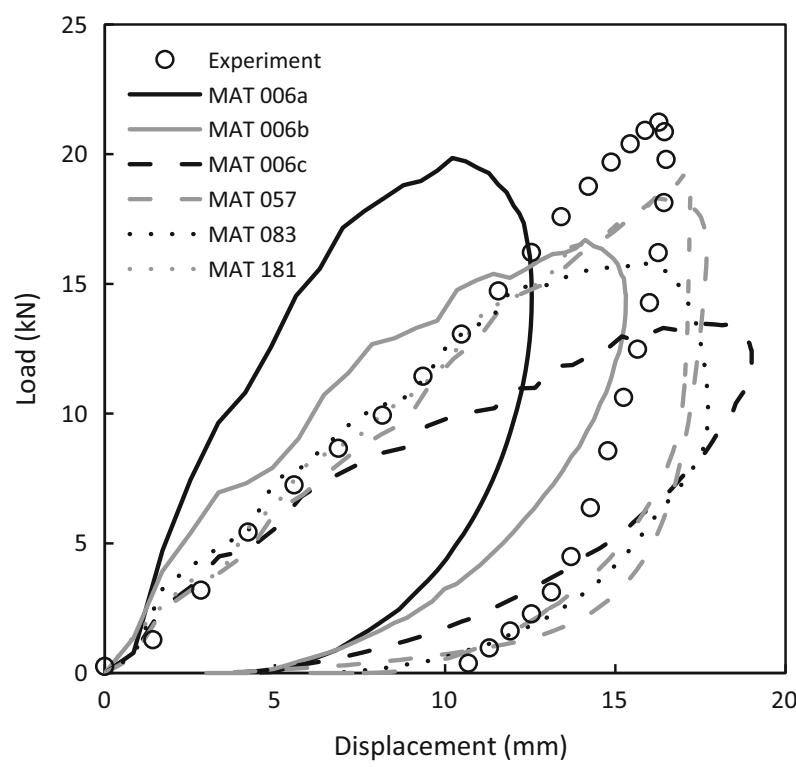

Fig. 5 Comparison of the experimental center of mass load displacement response with finite element material models

models have difficulty describing the observed load-displacement response, where viscoelastic models have the least agreement and MAT 181 agrees best.

The experimental coefficient of restitution, $e$, is compared with each numeric model in Fig. 6. While two of the viscoelastic models overpredict the coefficient of restitution, the remaining models are within $5 \%$ of the experimental values. The experimental ball stiffness is also compared with the finite element models in Fig. 6. Excluding the same two viscoelastic models with poor coefficient of restitution agreement (MAT 006b and MAT 006c), the models are within $25 \%$ of the experimental values. The results show that it is computationally more challenging to capture the ball stiffness than energy dissipation. This is a potentially serious drawback of computational models, as ball stiffness can have a large effect on 
Fig. 6 Comparison of the experimental cylindrical coefficient of restitution and stiffness with the finite element models

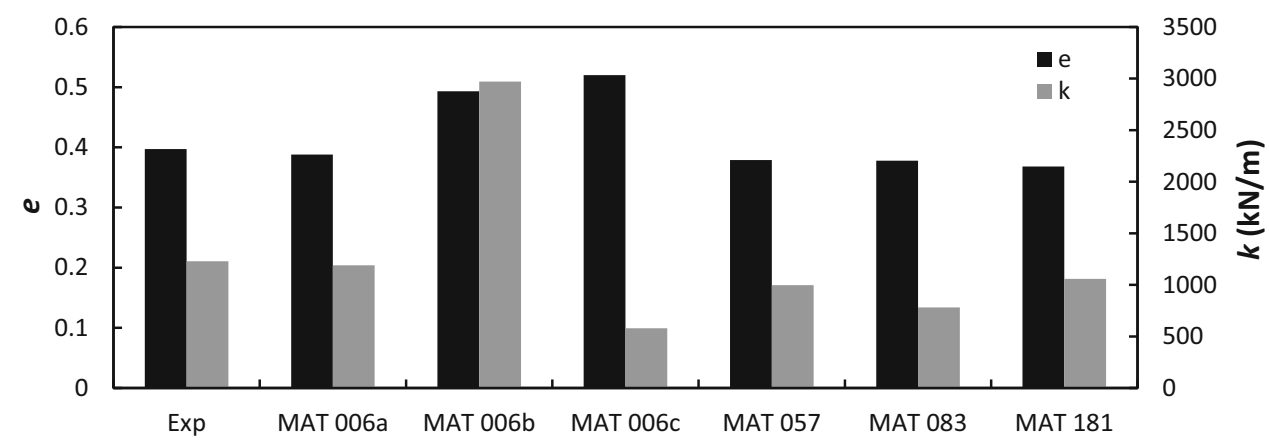

equipment performance through the so-called trampoline effect. Inaccuracy in ball stiffness can also affect personal impact and protective equipment simulations.

Video analysis of the impacts allows comparison of ball geometric features as a function of time. The experimental ball diameter in the direction parallel to the ball path, $D_{y}$ ( $y$ direction in Fig. 4), is compared with the finite element models in Fig. 7. The ball diameter is observed to decrease by a maximum of $18 \mathrm{~mm}$ and retains a $5 \mathrm{~mm}$ deformation after impact (the ball recovers completely within a few seconds of impact). All the models show good agreement with the experiment as the ball deformation commences. The MAT 006 models show the poorest agreement, overall. While MAT 006a showed good agreement with the coefficient of restitution and stiffness, it underestimates the change in ball diameter in Fig. 7. In contrast, the foam models provide a more accurate description of $D_{y}$, capturing the peak diameter change within the measurement accuracy of the video technique. Of all the models, MAT 181 came closest to describing the persistent deformation to within $3 \%$ after impact was complete.

The experimental ball diameter transverse to the ball path $\left(D_{x}\right.$ and $\left.D_{z}\right)$ increases during impact and is also presented in Fig. 7. The MAT 006 models exceed the observed diameter change, in some cases by large factors. MAT 006 relies on time-dependent response to describe energy dissipation. This material model can only capture the large energy dissipation for softballs using an artificially large bulk modulus. MAT 181 captures the deformation phase of $D_{x}$ (parallel to the cylinder axis) best, but does not describe the recovery after impact. The other foam models (MAT 057 and MAT 083) best capture $D_{y}$ (normal to the cylinder axis), while MAT 181 shows much higher deformation (comparable to MAT 006) than that observed experimentally.

The experimentally measured ball volume is compared with the finite element models in Fig. 8. Here, the volume of the MAT 006 models is nearly constant, consistent with the response of an incompressible material with a high bulk modulus. The volume of the foam material models compares more favorably with that of the experiment, where MAT 057 agrees best.

\section{Discussion}

Numeric simulations of ball impacts require a level of accuracy that has been difficult to achieve with the chosen material models. Viscoelastic and foam material models have the capabilities to control stiffness and energy dissipation needed to describe ball response. While viscoelastic models can be developed to control ball coefficient of restitution and stiffness, the material models chosen have been shown to have difficulty describing ball deformation. The chosen foam material models were able to more reliably capture the measured coefficient of restitution and ball stiffness, but also had difficulty describing deformation.

The response of each of the six material models considered in this work is compared in Fig. 9. All values are normalized with experiment and are averaged over the impact duration. To compare the load-displacement curves $(L-d)$, the ratio of the predicted and measured displacement at each data point (for the same load) was found. As in the other cases, the mean of these values is reported. While it is apparent from the figure that the foam material models match the experimental deformation $\left(D_{x}, D_{y}\right.$, and $\left.D_{z}\right)$ more closely than the viscoelastic models, compared to the other parameters, the error in ball diameter is relatively small. In addition, when the material model load-displacement response (obtained from the load cell) agreed with experiment, the measured deformations (using video) also tended to agree with those of the experiment. The material models had more difficulty describing the load-displacement, energy dissipation and stiffness.

The viscoelastic models chosen had large error describing ball volume, energy dissipation and ball stiffness. By comparison, the foam models had improved agreement with the experiment, particularly for the volume and load-displacement responses. While the foam models had more difficulty describing the ball coefficient of 

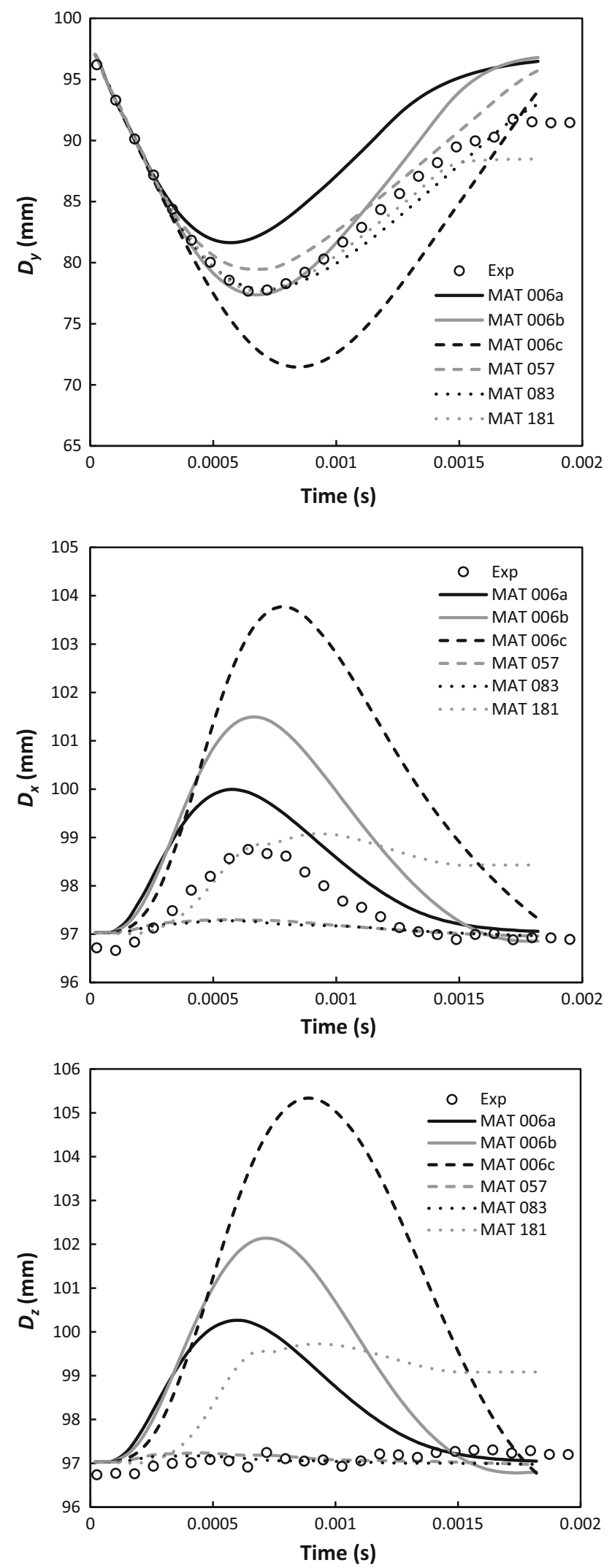

Fig. 7 Comparison of the experimental ball diameter along three normal directions with the finite element models

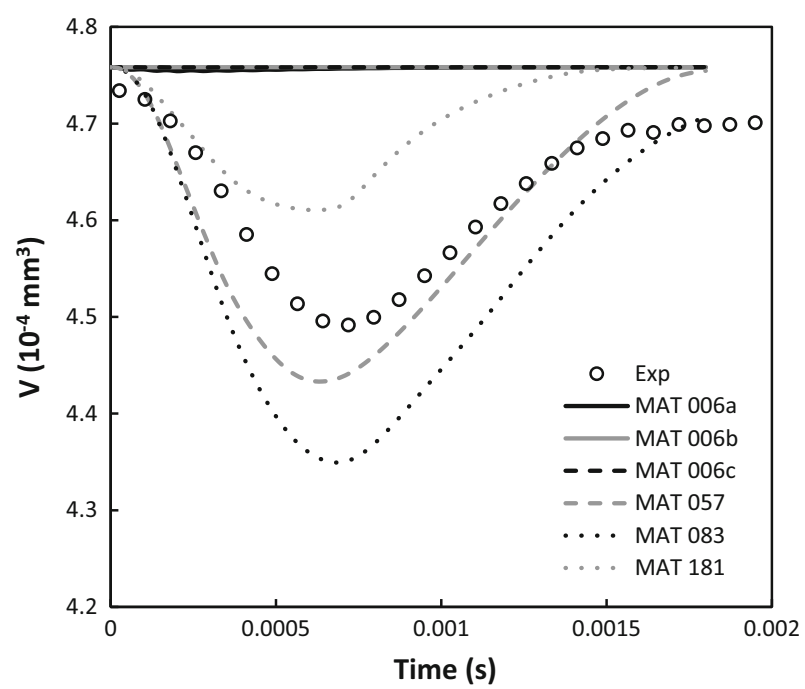

Fig. 8 Comparison of the experimental ball volume with the finite element models

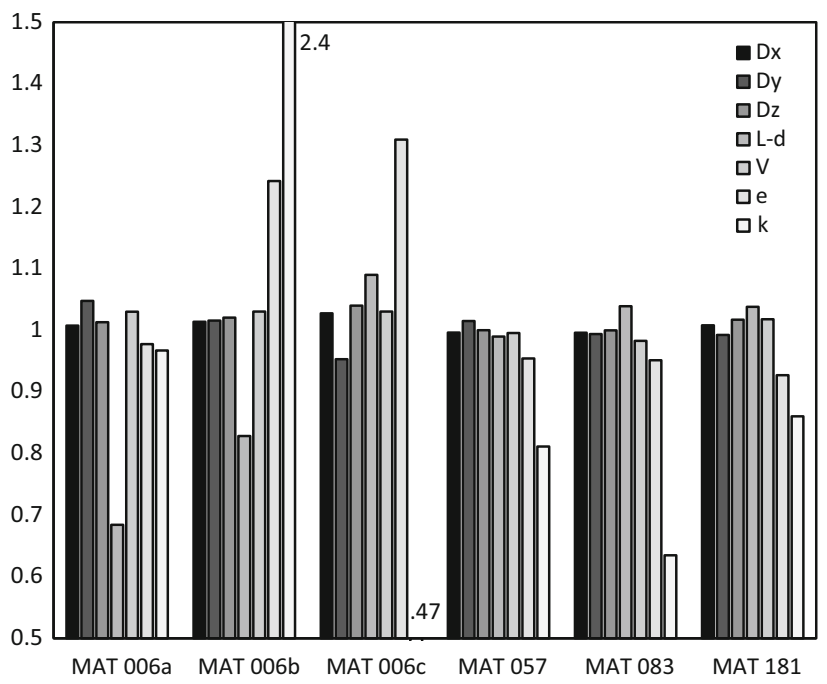

Fig. 9 Comparison of the six material models considered in this work. $D_{x}, D_{y}$ and $D_{z}$ represent the diameters according to the coordinate system defined in Fig. 4. $L-d$ Represents the loaddisplacement response. $V, e$ and $k$ are the ball volume, coefficient of restitution and stiffness, respectively. All values are normalized with the experiment and are averaged over the impact duration

restitution and stiffness, they compared more favorably with those of the experiment than the viscoelastic models. The foam material models describe the solid softball response more closely to experimental measurements than the viscoelastic models. The three foam materials models are relatively similar in their ability to describe experimental measurements; where one foam model was not universally superior to the others. This suggests that a fundamental material response of the rigid polyurethane 
foam may not be completely captured in these foam models. Further modeling effort on foam-constitutive models is needed to improve this comparison.

\section{Conclusions}

To quantify the ability of numeric models to describe sport ball impact response, video-based analysis to precisely model the 3D shape of the ball before, during and after impact is used. By comparing these 3D shapes to those predicted by the models, it has been demonstrated that foam-based material models describe ball deformation to within $1 \%$, whereas the viscoelastic models only come within $5 \%$. Both the viscoelastic and foam-constitutive models deviated more from experimental values when describing dissipated energy and stiffness than when describing deformations. When describing impact energy dissipation and ball stiffness, the viscoelastic models deviated from experiment by more than a factor of 2 , while the foam material models had up to $35 \%$ error. The measured ball deformation obtained from video analysis shows that foam material models are better at describing ball impacts involving large energy dissipation. The computer-vision algorithms have provided unprecedented quantitative geometric comparison between computer simulations and experimental observation. Video-based analysis has been shown to be an effective tool to validate computational models.

\section{References}

1. Bathke T (1998) Baseball Impact Simulation, Senior Thesis: Brown Univeristy

2. Mustone T, Sherwood J (2000) Using LS-DYNA to develop a baseball bat performance and design tool. Detroit, MI

3. Sandmeyer BJ (1994) Simulation of bat/ball impacts using finite element analysis, Master's Thesis: Oregon State University

4. Smith LV, Duris JG (2009) Progress and challenges in numerically modeling solid sports balls with application to softballs. J Sports Sci 27(4):353-360
5. Burbank SD, Smith LV (2012) Dynamic characterization of rigid polyurethane foam used in sports balls. Sports Eng Technol 226:77-85

6. Hendee SP, Greenwald RM, Crisco JJ (1998) Static and dynamic properties of various baseballs. J Appl Biomech 14(4):390-400

7. Ranga D, Strangwood M (2010) Finite element modelling of the quasi-static and dynamic behaviour of a solid sports ball based on component material properties, Vienna

8. Smith LV, Nathan AM, Duris JG (2010) A determination fo the dynamic response of softballs. Sports Eng 12(4):163-169

9. Allen T, Haake S, Goodwill S (2009) Comparison of a finite element model of a tennis racket to experimental data. Sports Eng 12(2):87-98

10. Choppin S, Goodwill S, Haake S (2010) Investigations into the effect of grip tightness on off-centre forehand strikes in tennis. Proc Inst Mech Eng Part P J Sports Eng Technol 224(4):249-257

11. Smith LV, Kensrud J (2014) Field and laboratory measurements of softball player swing speed. Sports Eng 17(2):75-82

12. Salzmann M, Moreno-Noguer F, Lepetit V, Fua P (2008) Closedform solution to non-rigid 3D surface registration. Marseille, FRANCE

13. Salzmann M, Fua P (2010) Deformable surface 3D reconstruction from monocular images, Morgan-Claypool

14. Perriollat M, Hartley R, Bartoli A (2011) Monocular templatebased reconstruction of inextensible surfaces. Int $\mathrm{J}$ Comput Vision 95(2):124-137

15. Ngo D, Ostlund J, Fua P (2016) Template-based monocular 3D shape recovery using Laplacian meshes. Pattern Anal Mach Intell (PAMI) 38(1):172-187

16. Bryson A, Smith L (2010) Impact response of sports materials. Austria, Vienna

17. Nathan AM, Smith LV, Faber WL (2011) Reducing the effect of the ball on bat performance measurements. Sports Technol 4(1):19-28

18. Chang F (1995) Development of LSDYNA3D Foam Material Type 83

19. Benson DJ, Kolling S, Bois PAD (2006) A simplified approach for strain-rate dependent hyperelastic materials with damage. In: 9th International LS-DYNA Users Conference, vol 15. Dearborn, MI

20. Nevins D, Smith L (2014) Methods for Modeling Solid Sports Ball Impacts. In: 13th International LS-DYNA Conference, Dearborn

21. Nevins D,Smith L (2013) Influence of ball properties on simulated ball-to-head impacts. In: 6th Asia-Pacific Conference on Sports Technology, The Impact of Technology on Sports, Hong Kong 